\title{
Seleção de um referencial teórico e análise bibliométrica da gestão do conhecimento e design na agricultura familiar ${ }^{[1]}$
}

Selection of a Theoretical Reference and Bibliometric Analysis of Knowledge Management and Design in Family Agriculture

Marco Antonio Weiss ${ }^{[2]}$, Luiz Fernando Gonçalves de Figueiredo $^{[3]}$, Gregório Jean Varvakis Rados ${ }^{[4]}$ 
Resumo: A agricultura familiar possui hoje um importante papel social e econômico, sendo responsável por $70 \%$ da produção dos alimentos consumidos no Brasil. Esta pesquisa visa estabelecer uma relação entre os temas Agricultura Familiar, Gestão do Conhecimento e Design através de uma análise bibliométrica de artigos publicados e disponíveis na base de dados Web Of Science, tendo como objetivo identificar o avanço de pesquisas que relacionam tais temas, observando as possíveis contribuições da Gestão do Conhecimento e do Design para empreendimentos de Agricultura Familiar. Neste artigo, de caráter exploratório e descritivo, os resultados mostram um crescimento no número de pesquisas que relacionam dois desses termos, mas ainda são poucas as publicações que associam os três termos.

Palavras Chave: Design. Gestão do Conhecimento. Agricultura Familiar.

Abstract: Family agriculture today has an important social and economic role, being responsible for $70 \%$ of the production of food consumed in Brazil. This research aims to establish a relationship between the themes "family agriculture", knowledge management "and" Design "through a bibliometric analysis of published articles and available in the Web of Science database, aiming to identify the advance of research that relate the three themes, observing the possible contributions of knowledge management and Design in the development of family farming endeavors. In this article, of an exploratory and descriptive nature, quantitative indicators are presented about the results obtained that will serve as a foundation for future studies.

Keywords: Design. Knowledge Management. Family Agriculture.

\section{INTRODUÇÃO}

Nos últimos anos o modelo agrícola produtivista tem produzido efeitos negativos nas esferas econômica, social e ambiental, fazendo com que seja necessário repensar os modelos tecnológicos dominantes e trazendo para debate novas formas de produção agrícola, diferentes funções para a agricultura e mudanças nas relações entre o homem e natureza (MIOR et al, 2014).

Carreira (2017) discorre que o desenvolvimento da sociedade está profundamente relacionado ao desenvolvimento do homem como um ser espiritual em um processo de busca por aprendizado e evolução nessa condição e, ainda, procurando suprir suas necessidades afetivas, materiais e espirituais. Em busca de atender a essas necessidades, o homem, por vezes, sucumbe aos comportamentos padrões impostos por uma sociedade enraizada numa cultura de valores em que 
o 'TER' se sobressai ao 'SER'. Como protagonista da história social, o homem é o centro de todas as transformações culturais, educacionais e políticas em todas as épocas.

Nesse contexto, Hickel (2015) acrescenta que o papel do designer, se exercido sob o mesmo sistema antropocêntrico de valores, limita-se em como tornar "verde" os objetos de uma cultura essencialmente antropocêntrica. O design é hoje responsável por práticas e patologias insustentáveis do ponto de vista ecológico e ambiental. O design opera os processos de transformação do natural em uma miríade de sistemas e objetos e que podem promover ou danificar as condições ecológicas e assim, empobrecer ou enriquecer o viver.

$\mathrm{Na}$ presente pesquisa foram utilizados os termos "Agricultura Familiar", "Gestão do Conhecimento" e "Design" para investigar o panorama da produção científica atual, bem como se dá a interação dessas diferentes áreas do conhecimento entre si. Gil define que "o objetivo fundamental da pesquisa é descobrir respostas para problemas mediante o emprego de procedimentos científicos" (2014, p. 26.). Para tanto, esta pesquisa tem como objetivo identificar o avanço de pesquisas que relacionam os três temas, observando as possíveis contribuições da Gestão do Conhecimento e do Design no desenvolvimento de empreendimentos de Agricultura Familiar através de uma análise bibliométrica de artigos publicados e disponíveis na base de dados Web Of Science entre os anos de 2012 e 2018.

Para fim de atingir aos objetivos propostos, este artigo segue estruturado em cinco seções, a primeira sendo a que apresenta esta introdução, a segunda composta pela revisão da literatura, onde os conceitos chave são fundamentados, a terceira pelos procedimentos metodológicos da pesquisa, a quarta pela análise bibliométrica dos resultados obtidos nas buscas e, por fim, a quinta seção apresenta as conclusões da pesquisa empreendida. 


\section{REVISÃO DA LITERATURA}

Esta seção destina-se à fundamentação teórica das palavras-chave que foram utilizadas para a seleção dos artigos que deram subsídio para a construção do estudo: Agricultura Familiar, Gestão do Conhecimento e Design.

\section{Agricultura Familiar}

O engajamento coletivo no meio rural proporciona a formulação de estratégias para incrementar e diversificar as rendas agrárias, por meio da organização da produção, conquista de novos mercados, estratégias para a inclusão social e o direito à participação cidadã na construção de alternativas orientadas às necessidades locais (COSTABEBBER; MOYANO, 2000).

Já o AIAF (2014) define que "a agricultura familiar inclui todas as atividades agrícolas de base familiar, envolvendo produções agrícola, florestal, pesqueira, pastoril e aquícola que são gerenciadas e operadas por uma família e, predominantemente, dependentes de mão-de-obra familiar".

Lamarche (1993), por sua vez, identifica três perfis de agricultores familiares: (i) modelo familiar: são aqueles que não têm por finalidade a reprodução enquanto unidade de produção, mas sim a reprodução familiar; (ii) modelo de subsistência: o interesse dos agricultores está apenas na sobrevivência da família; (iii) modelo de empreendimento agrícola: são motivados por uma exploração agrícola organizada com base no trabalho assalariado, orientada para obtenção de lucro.

A agricultura familiar é responsável por aproximadamente $80 \%$ dos alimentos consumidos no mundo, sendo também o principal responsável pela comida que chega às mesas das famílias brasileiras, respondendo por cerca de $70 \%$ da produção nacional (FAO, 2014). No estado de Santa Catarina, cerca de $90 \%$ dos estabelecimentos rurais são classificados como agricultura familiar, áreas com no máximo 50 hectares (IBGE, 2009). 


\section{Gestão do Conhecimento}

A informação e o conhecimento só têm valor quando ultrapassam o campo do pensar para o campo do agir. Assim, entende-se que, no momento atual, o conhecimento é um dos fatores que garante competitividade. Mas, esse conhecimento é validado se for compartilhado por toda a organização e transformado em conhecimento organizacional. Desse modo, quanto mais o conhecimento for compartilhado maiores serão as oportunidades de aprendizagem dos colaboradores (CARREIRA, 2017). Dotsika e Patrick (2013) explicam que a Gestão do Conhecimento dentro das organizações se expressa na necessidade de aumentar as vendas, aprimorar as práticas de trabalho e tomada de decisão, reduzir o tempo de desenvolvimento, melhorar serviços ao cliente, capacitar os funcionários e inovar. Bassi (1999), por sua vez, define a Gestão do Conhecimento como o processo de criação, captura e utilização do conhecimento com o objetivo de melhorar o desempenho organizacional. Para Carreira (2017), no processo de implementação da Gestão do Conhecimento, é preciso criar mecanismos que atendam às necessidades das pessoas e proporcionar um ambiente propício à criação e aprendizagem.

A Gestão do Conhecimento é um processo de transformação situado entre a economia dos recursos naturais e a do capital intelectual. E tem como um dos seus principais objetivos considerar o conhecimento como um ativo para a empresa, sendo necessário promover sua permanência para que gere riqueza. Assim, é imprescindível que se invista em pessoas, criando uma cultura voltada para a criação e retenção do conhecimento, para que tais ativos possam contribuir no processo de crescimento e inovação. (TENG; SONG, 2011).

\section{Design}

Para criar artefatos com maiores chances de influenciar o comportamento, designers e pesquisadores identificaram a necessidade de entender melhor a formação dos hábitos e escolhas dos usuários. Tal condição motivou a aproximação 
do design a preceitos teóricos da psicologia, proporcionando a emergência de um amplo espectro de abordagens reconhecido como design para mudança de comportamento. O propósito deste campo consiste na compreensão mais apurada das motivações e desejos dos indivíduos e na tradução deste conhecimento em estratégias para estimular atitudes em benefício social e ambiental (LOCKTON et al., 2010).

Coelho (2011) define que a função do design é dar forma a artefatos, levando em consideração um projeto elaborado previamente com um escopo específico. $O$ trabalho do designer envolve também questões de uso, função, produção, mercado, utilidade, qualidade formal e estética, buscando equacionar, sistêmica e simultaneamente, fatores sociais, culturais, antropológicos, ecológicos, ergonômicos, tecnológicos e econômicos.

Em resposta à natureza ampla e multidisciplinar do design para mudança de comportamento, Niedderer et al. (2014) elaboraram um mapeamento de suas abordagens mais relevantes. $O$ estudo analisou também de que forma tais perspectivas associam-se às teorias comportamentais, identificando quatro áreas nas quais os projetos deste campo são potencialmente aplicados: tecnologia persuasiva, design para o comportamento sustentável, design social e contra o crime, e design para o bem-estar.

\section{PROCEDIMENTOS METODOLÓGICOS}

Este estudo segue como metodologia um levantamento exploratório de artigos publicados na base de dados Web of Science. Gil (2014) explica que a finalidade da pesquisa exploratória é desenvolver, esclarecer e modificar conceitos e ideias, de modo a formular problemas mais precisos ou hipóteses pesquisáveis para estudos futuros. As pesquisas exploratórias podem ainda ser a primeira etapa de uma investigação mais ampla e ter como produto final um problema mais esclarecido.

A busca foi realizada sob o Proxy da instituição UFSC acessando a base Web of Science no Portal de Periódicos da CAPES no dia 26 de fevereiro de 2018. Com o objetivo de rea- 
lizar um estudo bibliométrico descritivo, foram usadas as palavras-chave: "Agricultura Familiar" ("Family Agriculture"); "Gestão do Conhecimento" ("Knowledge Management") e ("Design") e suas associações entre si, com o filtro do período dos anos entre 2012 e 2018 e selecionados apenas artigos. Não foram utilizados filtros de área ou idioma.

A pesquisa se iniciou buscando artigos que tivessem a palavra ("Family Agriculture") entre suas palavras-chave supracitadas, em seguida o mesmo foi realizado com as palavras ("Knowledge Management") e ("Design"), verificando quais áreas do conhecimento reconhecidas pela CAPES mais se relacionavam com os termos e o crescimento na quantidade de publicações. Em seguida, foram buscados artigos que associassem em suas palavras-chave: ("Family Agriculture" AND "Knowledge Management"); ("Knowledge Management" AND "Design"); ("Knowledge Management" AND "Design") e, por fim, foram pesquisados artigos que associassem os três termos: ("Family Agriculture" AND "Knowledge Management" AND "Design"). Para afunilar mais a pesquisa, buscou-se pela quantidade de publicações exclusivas da área de Ciências Sociais Aplicadas. Para finalizar, foram observadas as cinco principais fontes de publicações de artigos de cada associação de termos. A Figura 1 ilustra os procedimentos realizados na presente pesquisa.

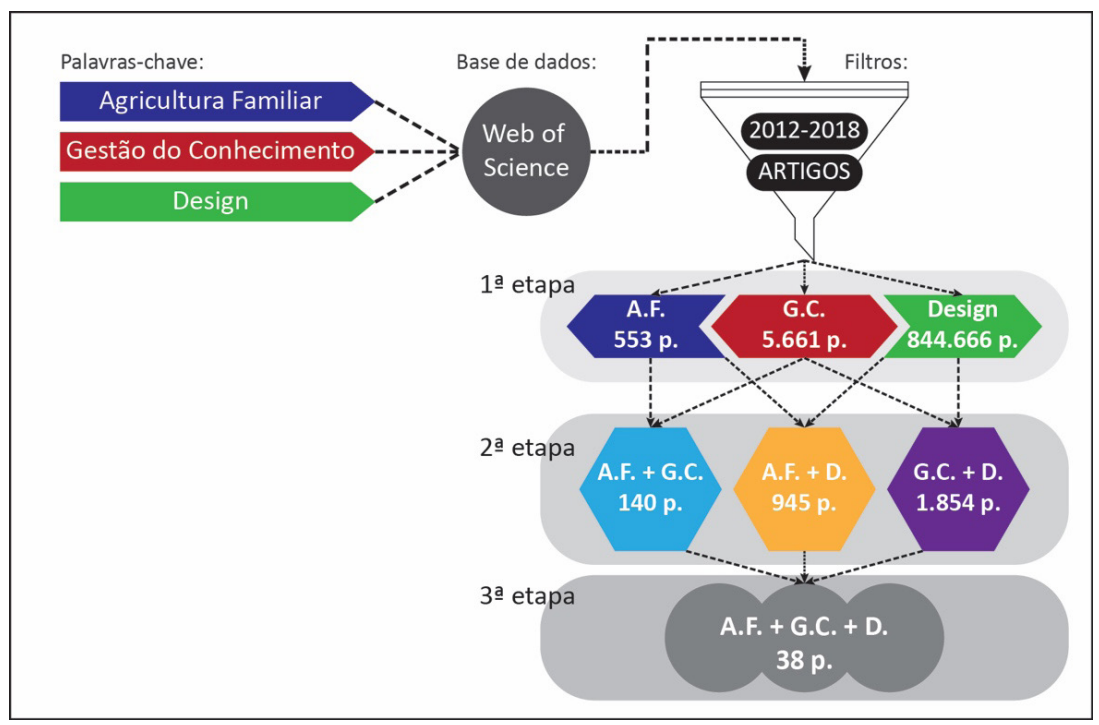

Figura 1 - Diagrama passos da pesquisa Fonte: Elaborado pelos autores (2018) 


\section{RESULTADOS E DISCUSSÕES}

A definição das palavras-chave utilizadas na seleção dos artigos respeitou o parâmetro dos três eixos da pesquisa, sendo que o primeiro eixo envolve as palavras-chave relacionadas à Agricultura Familiar, o segundo a Gestão do Conhecimento e o terceiro eixo o Design. As palavras-chave foram testadas na base de dados para verificar quais termos mais se relacionam com as pesquisas encontradas e, assim, ampliar o alcance da busca.

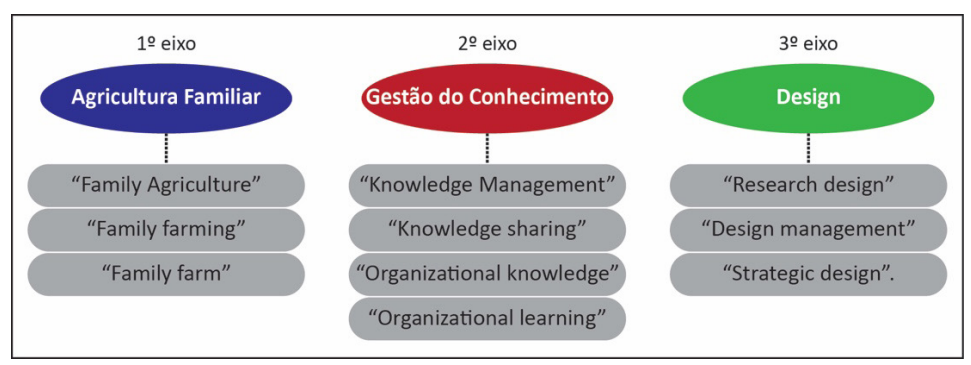

Figura 2 - Eixos temáticos. Fonte: Elaborado pelos autores (2018).

A figura 2 apresenta os três eixos da pesquisa com as palavras-chave associadas a cada um. Os termos relativos ao terceiro eixo temático se associam a palavra "Design" com o interesse e área de pesquisa dos pesquisadores, a Gestão de Design.

Deu-se início à pesquisa buscando pelo tópico: "Agricultura Familiar". Foram encontradas 553 publicações até a data da pesquisa, porém, entre 2015 e 2017 é que se concentra o maior número de publicações, demonstrando o aumento do interesse em se discutir a questão da Agricultura Familiar. $\mathrm{O}$ gráfico a seguir (Figura 3) apresenta a evolução da quantidade de artigos por ano. 


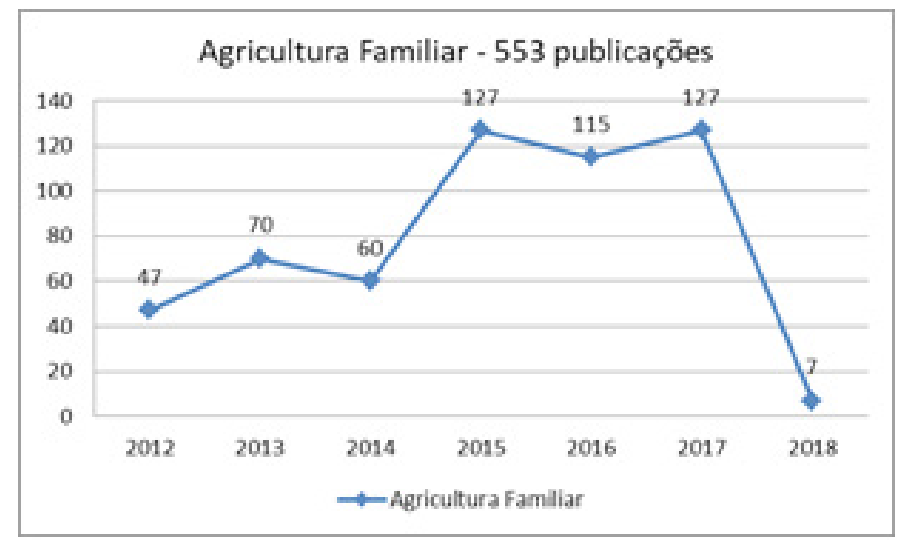

Figura 3 - Publicações por ano, Agricultura Familiar. Fonte: Elaborado pelos autores (2018).

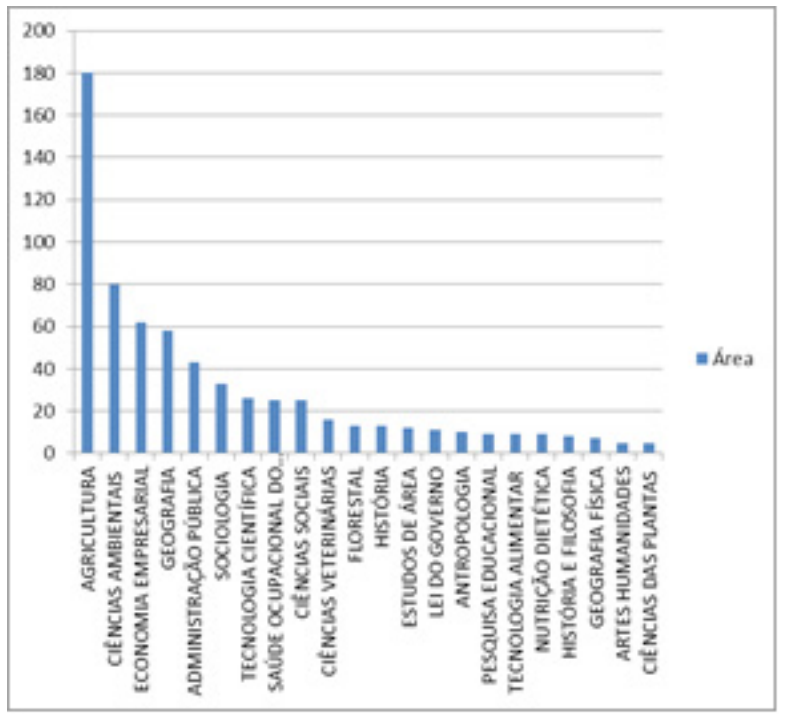

Figura 4 - Área do conhecimento, Agricultura Familiar. Fonte: Elaborado pelos autores (2018).

Com relação à área do conhecimento a qual as publicações se enquadram, a grande maioria dos estudos envolve a Agricultura, sendo 180 das 553 publicações encontradas. Em seguida as três áreas mais abordadas foram Ciências Ambientais, Economia Empresarial e Geografia, como mostrado na Figura 4.

Em seguida buscou-se os resultados ao tópico: “Gestão do Conhecimento". Este tópico apresentou 5.661 publicações como resultado. Este tópico também apresentou um pico com maior número de publicações no período entre 2015 e 
2017, como mostrado na Figura 5, nesse período foram publicados 3.403 artigos que abordaram esse assunto dos 5.661 encontrados. A área do conhecimento com maior destaque foi a Economia Empresarial, com 2.531 publicações das 3.403, seguida da Ciência da Informação, Ciência da Computação e Engenharia (Figura 6).

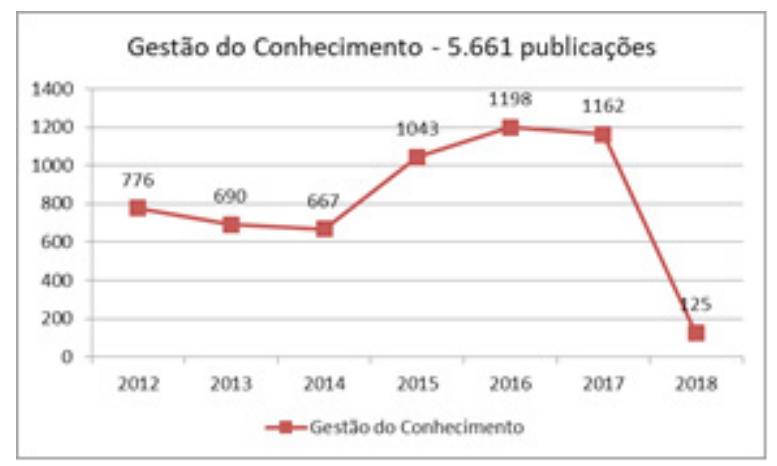

Figura 5 - Publicações por ano, Gestão do Conhecimento. Fonte: Elaborado pelos autores (2018).

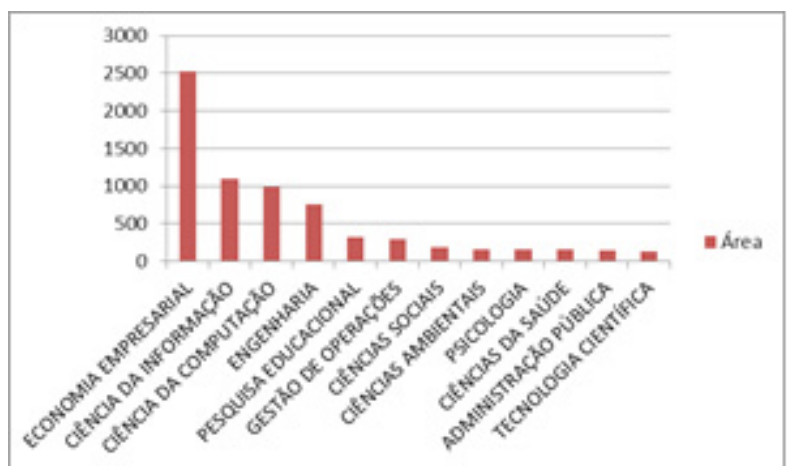

Figura 6 - Área do Conhecimento, Gestão do Conhecimento. Fonte: Elaborado pelos autores (2018).

O terceiro tópico pesquisado, "Design", foi o que apresentou maior número de resultados, com 844.666 publicações no período, se apresentando em constante crescimento desde 2012 (Figura 7). A Engenharia é a área com maior enfoque nas publicações, com 211.568 artigos nessa área, em sequência estão a Química, Ciência da Computação e Ciência dos Materiais (Figura 8). 


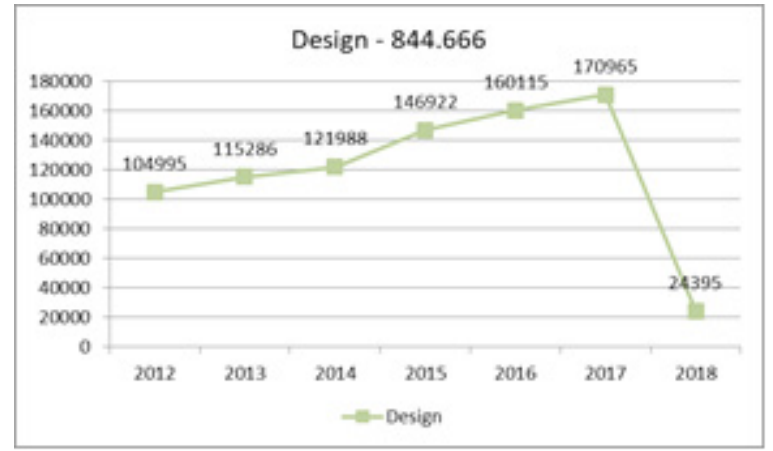

Figura 7 - Publicações por ano, Design. Fonte: Elaborado pelos autores (2018).

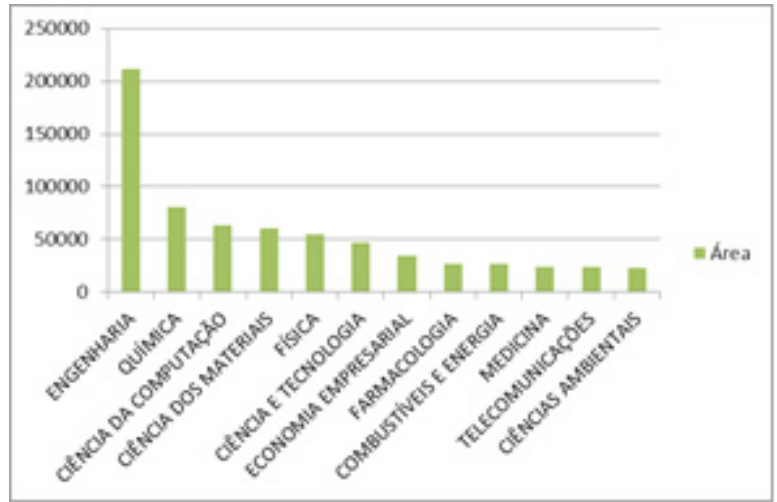

Figura 8 - Área do Conhecimento, Design. Fonte: Elaborado pelos autores (2018).

A etapa seguinte das buscas na base de dados se refere à associação dos termos entre eles. Desse modo verificou-se quais artigos encontrados sobre "Agricultura Familiar" também apresentavam o termo "Gestão do Conhecimento". Usando este tópico, a base de dados Web of Science apresentou 140 resultados, estes resultados descrevem um discreto crescimento do número de publicações desde 2013 (Figura 9). As áreas do conhecimento com mais publicações com a associação dos termos "Agricultura Familiar" + "Gestão do Conhecimento" são a da Agricultura e da Ciência Ambiental (Figura 10). 


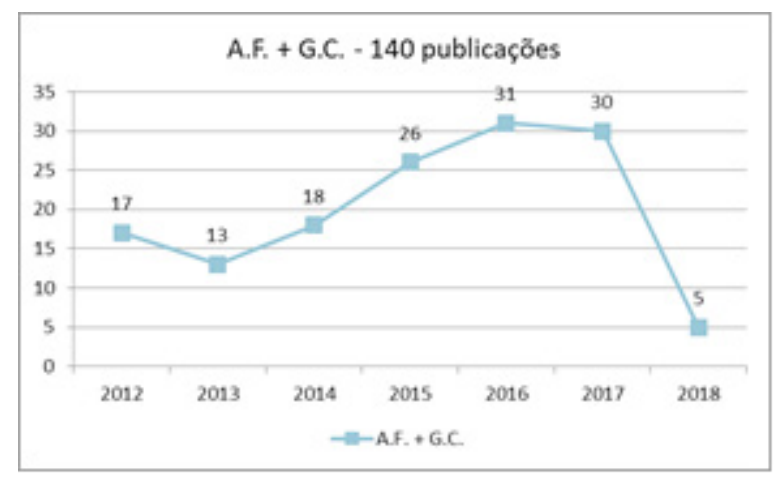

Figura 9 - Publicações por ano, Agricultura Familiar + Gestão do Conhecimento. Fonte: Elaborado pelos autores (2018).

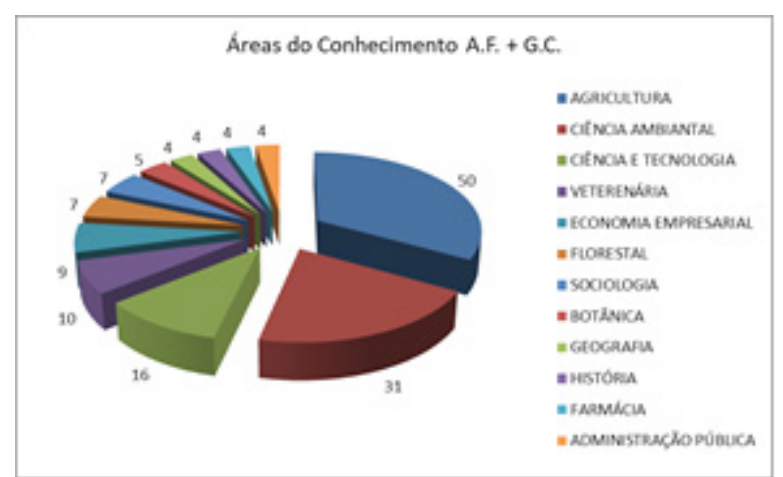

Figura 10 - Área do conhecimento, Agricultura Familiar + Gestão do Conhecimento. Fonte: Elaborado pelos autores (2018).

A Figura 11 identifica as principais fontes das publicações encontradas, ou seja, os periódicos onde os artigos foram publicados, neste tópico o periódico com mais publicações foi o Crop Protection. O país com o maior número de publicações que associam a A.F. com a G.C. foi o Brasil, com 27 publicações nesse período (Figura 12). 


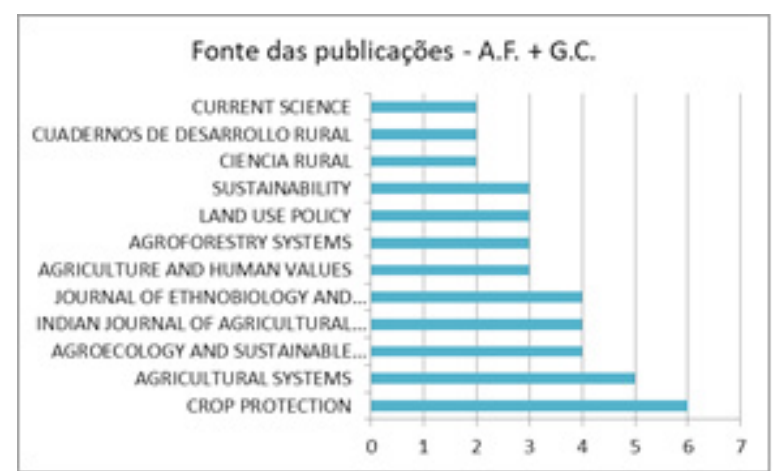

Figura 11 - Fonte publicações, Agricultura Familiar + Gestão do Conhecimento. Fonte: Elaborado pelos autores (2018).

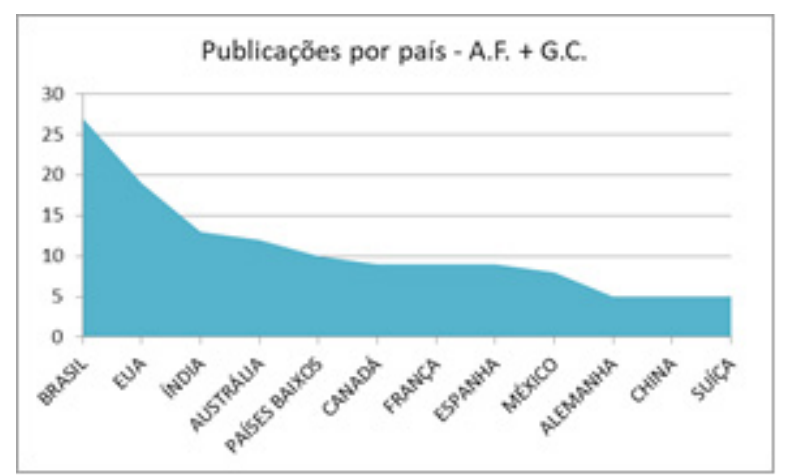

Figura 12 - Publicações por país, Agricultura Familiar + Gestão do Conhecimento. Fonte: Elaborado pelos autores (2018).

Na sequencia, a segunda associação feita foi com os termos "Gestão do Conhecimento" + "Design". Com este tópico foram encontrados 1.854 artigos. A Figura 13 mostra que entre os anos de 2012 e 2014 houve um pequeno declínio no número de publicações com esses termos e entre 2014 e 2017 o número de publicações voltou a crescer. Com relação às áreas do conhecimento, a Economia Empresarial e a Ciência da Informação foram as com mais publicações nesse período, como representado na Figura 14. 


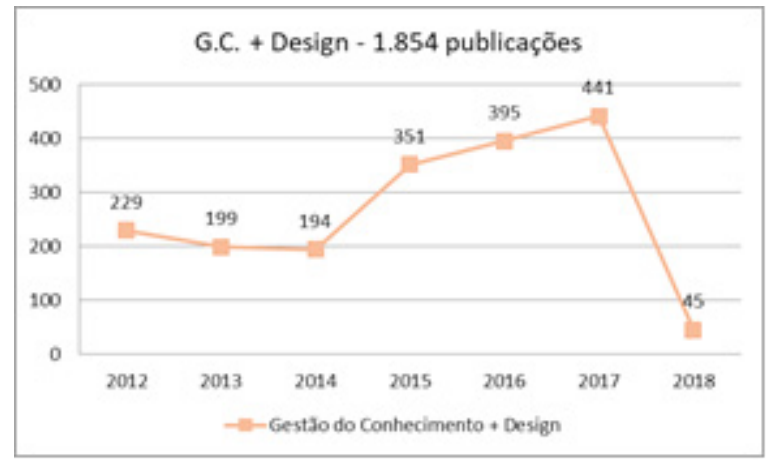

Figura 13 - Publicações por ano, Gestão do Conhecimento + Design. Fonte: Elaborado pelos autores (2018).

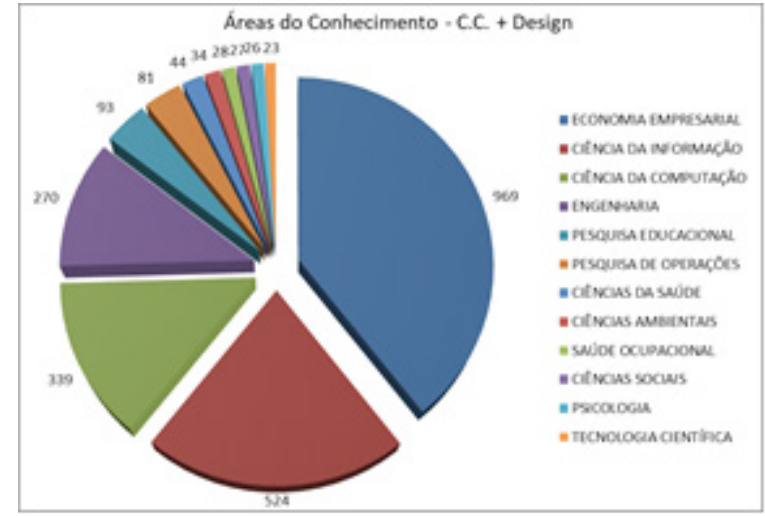

Figura 14 - Área conhecimento, Gestão do Conhecimento + Design. Fonte: Elaborado pelos autores (2018).

O periódico Journal of Knowledge foi a fonte encontrada com o maior número de publicações que associam G.C. e Design, apresentando 285 publicações, uma grande diferença da fonte com o segundo maior número, a Learning Organization, com 55 publicações no período (Figura 15). A Figura 16 mostra os EUA como sendo o país com o índice mais elevado de publicações, 319. 


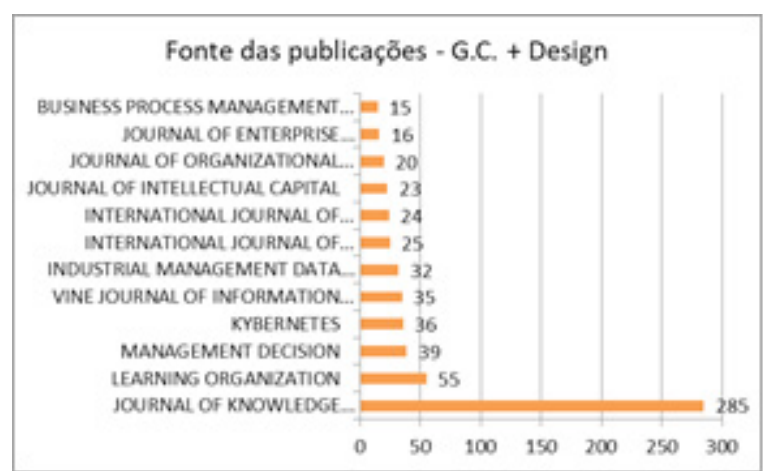

Figura 15 - Fonte publicações, Gestão do Conhecimento + Design. Fonte: Elaborado pelos autores (2018).

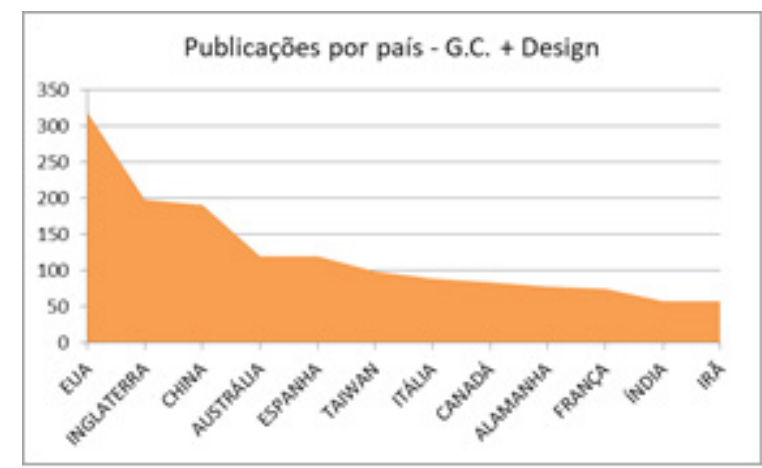

Figura 16 - Publicações por país, Gestão do Conhecimento + Design. Fonte: Elaborado pelos autores (2018).

A associação das palavras-chave "Agricultura Familiar" + "Design" buscou 945 resultados (Figura 17), com um singelo crescimento no número de publicações a partir de 2013 a 2017. As áreas de conhecimento as quais os estudos se enquadram aparecem na Figura 18, sendo a Agricultura a área com o maior número, 697 das 945 publicações, o restante das áreas abordadas não apresentaram diferenças expressivas nesse sentido. 


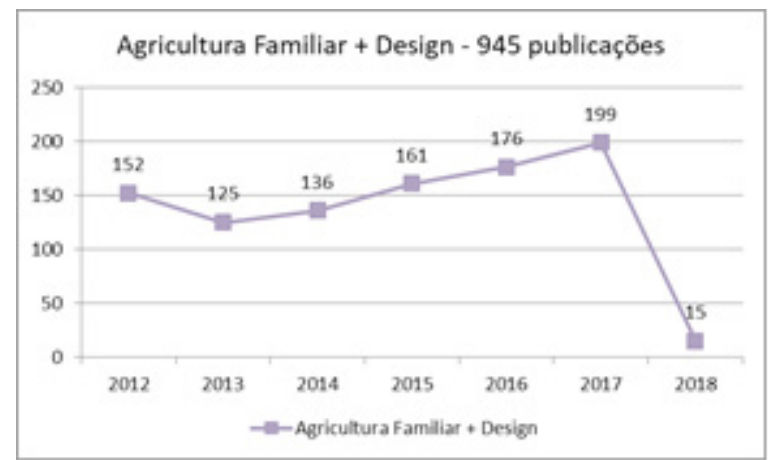

Figura 17 - Publicações por ano, Agricultura Familiar + Design. Fonte: Elaborado pelos autores (2018).

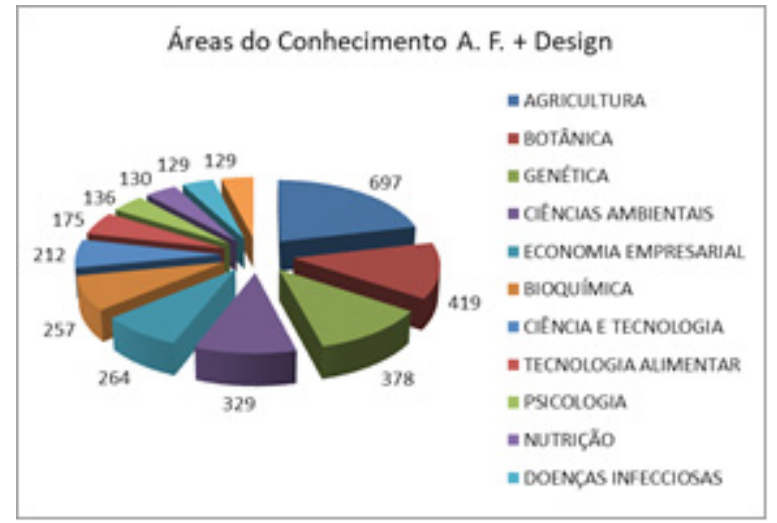

Figura 18 - Área conhecimento, Agricultura Familiar + Design. Fonte: Elaborado pelos autores (2018).

As três fontes com mais artigos publicados com os termos A.F. + Design são Plos One, Public Health Nutrition e Euphytica (Figura 19). O país com mais estudos nessa área foi EUA, 233 artigos, seguido do Brasil, 121 e China, 101 (Figura 20). 


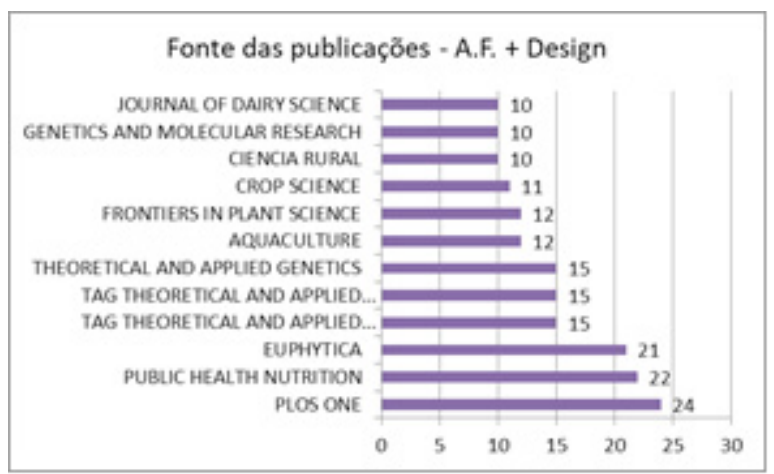

Figura 19 - Fonte publicações, Agricultura Familiar + Design. Fonte: Elaborado pelos autores (2018).

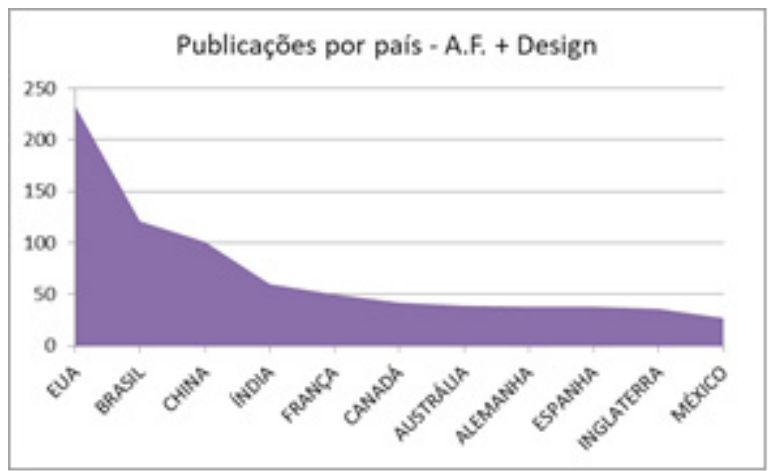

Figura 20 - Publicações por país,Agricultura Familiar + Design. Fonte: Elaborado pelos autores (2018).

Por fim, na útima etapa da pesquisa, foram buscados estudos que associassem os três tópicos "Agricultura Familiar" + "Gestão do Conhecimento" + "Design". Essa busca na Web of Science apresentou apenas 38 artigos publicados entre 2012 e 2018, sendo 2012 e 2017 os anos com mais publicações (Figura 21). Assim como nas outras buscas, as áreas com maiores números de publicações com essa associação de termos foram Agricultura e Ciências Ambientais (Figura 22). 


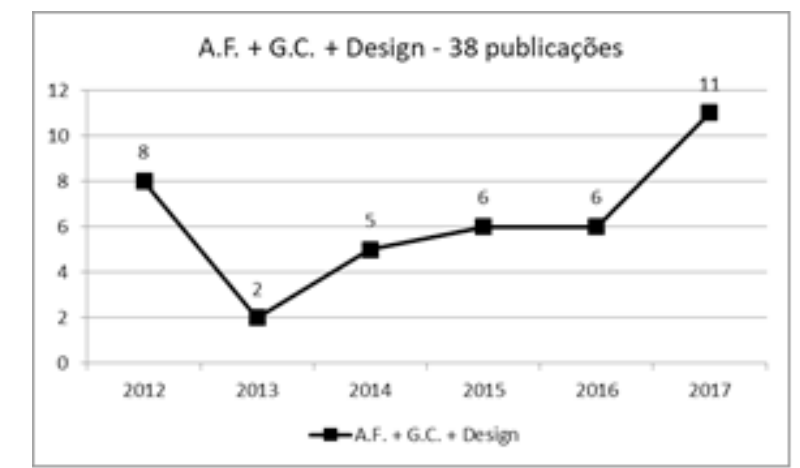

Figura 21 - Publicações por ano, Agricultura Familiar + Gestão do Conhecimento + Design. Fonte: Elaborado pelos autores (2018).

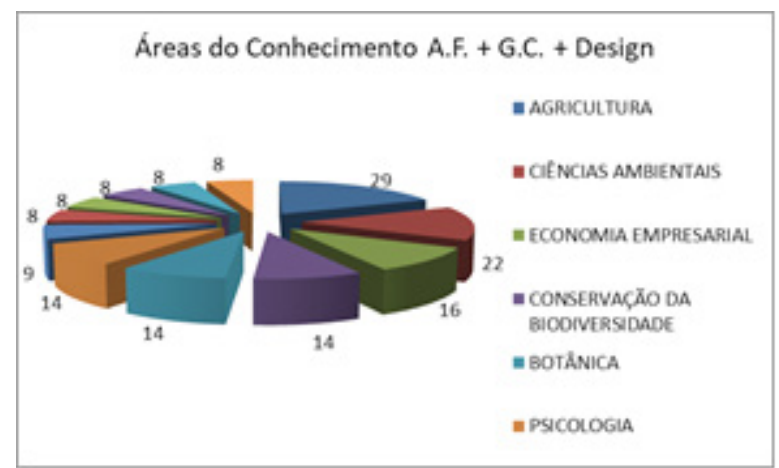

Figura 22 - Área conhecimento, Agricultura Familiar + Gestão do Conhecimento + Design. Fonte: Elaborado pelos autores (2018).

Por este tópico ter apresentado um número pequeno de resultados, os índices das análises também são baixos, exibindo pouca diferença entre uma colocação e outra. Os periódicos que se destacam nesse sentido foram: Agroforestry Systems, Indian Journal of Agricultural Sciences e Journal of Ethnobiology and Thnomedicine, os demais periódicos não aparecem no gráfico por apresentarem apenas uma publicação cada (Figura 23). Os países que realisaram os estudos encontrados em maior número foram EUA, com 8 estudos, seguido por Canadá, França e Índia, com 5 estudos cada um (Figura 24). 


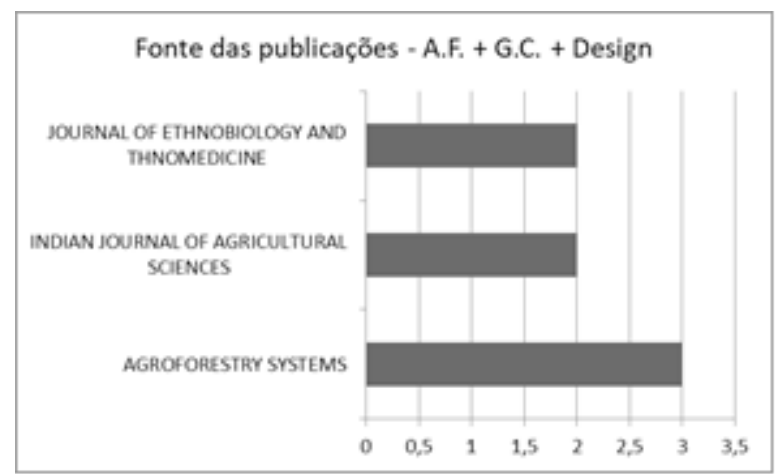

Figura 23 - Fonte publicações, Agricultura Familiar + Gestão do Conhecimento + Design. Fonte: Elaborado pelos autores (2018).

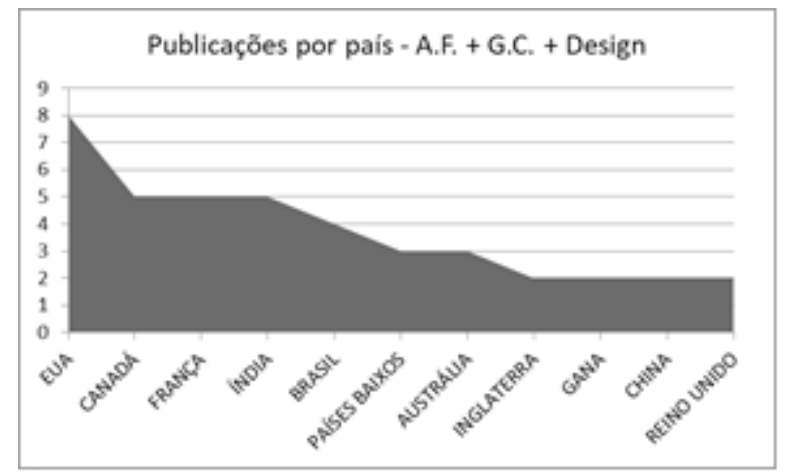

Figura 24 - Publicações por país, Agricultura Familiar + Gestão do Conhecimento + Design. Fonte: Elaborado pelos autores (2018).

Em contrapartida aos resultados anteriores, observa-se que o número de artigos encontrados nessa última etapa de análise, que se relacionam com as três áreas da pesquisa, apresentou um número bastante inferior ao encontrado nas etapas anteriores, sendo 140 artigos que relacionam Agricultura Familiar + Gestão do Conhecimento, 1.854 artigos de Gestão do Conhecimento + Design, 945 artigos de Agricultura Familiar + Design 


\section{CONSIDERAÇÕES FINAIS}

Esta pesquisa buscou evidenciar estudos e publicações nas áreas de "Agricultura Familiar", "Gestão do Conhecimento" e "Design", bem como estudos que associam os termos Agricultura Familiar + Gestão do Conhecimento, Gestão do Conhecimento + Design e Agricultura Familiar + Design. Dessa forma, constatou-se que publicações com esses temas se apresentam em crescimento, principalmente a partir de 2014. Vale ressaltar que as pesquisas foram realizadas considerando o ano presente, 2018, que, por estar ainda no primeiro trimestre, apresenta poucos resultados, porém mesmo este ano estando no início, já apresentou resultados em todas as buscas, significando que perdura o interesse por estudos em tais áreas.

Em um contexto geral, com relação às áreas do conhecimento em que as pesquisas estão inseridas, a Agricultura, Economia Empresarial e Engenharia são as áreas com maior destaque em número de artigos publicados. EUA e Brasil são os países que lideram o maior número de pesquisas envolvendo os termos buscados nessa análise. A etapa da pesquisa que apresentou um número bastante reduzido de estudos foi a que buscou por publicações que associassem os três termos: Agricultura Familiar, Gestão do Conhecimento e Design, com 38 publicações nos últimos 5 anos, demonstrando um campo oportuno para estudos interdisciplinares.

O caráter exploratório desta pesquisa, buscou proporcionar um meio viável para o desenvolvimento local sustentável, de forma que os resultados aqui obtidos poderão servir como base para estudos futuros que objetivem buscar ou desenvolver meios para que designers, munidos de seu know-how, junto das contribuições da Gestão do Conhecimento, possam auxiliar empreendimentos de agricultura familiar a se desenvolverem estrategicamente. 


\section{REFERÊNCIAS BIBLIOGRÁFICAS}

AIAF - Ano Internacional da Agricultura Familiar 2014. O que é agricultura familiar? Organização das Nações Unidas para Agricultura e Alimentação, 2014. (http://www.fao.org/ family-farming-2014/home/what-is-family-farming/pt/)

BASSI, L. Harnessing the power of intellectual capital. The Knowledge Management Yearbook 19992000. Boston: Butterworth Heinemann, 1999.

CARREIRA, S. S. Diretrizes para práticas de gestão do conhecimento nas organizações de economia de comunhão à luz do perfil do empreendedor social. Dissertação (Mestrado). Florianópolis: Universidade Federal de Santa Catarina, 2017.

COELHO, L. A. L. (Org.). Conceitos-chave em design. Rio de Janeiro: Ed. PUC-Rio. Novas Ideias, 2011.

COSTABEBER, J. A.; MOYANO, E. Transiçao agroecológica e ação social coletiva. Agroecologia e Desenvolvimento Rural Sustentável, v. 1, n. ${ }^{\circ}$, p. 50-60, out./dez. 2000.

DOTSIKA, K; PATRICK, K. Collaborative KM for

SMEs: a framework evaluation study. Information

Technology \& People, v. 26, n. ${ }^{\circ}$ 4, p. 368-382, 2013.

FAO. Colocar os agricultores familiares em primeiro para erradicar a fome. Food and Agriculture Organization of the United Nations, 2014. (http://www. fao.org/news/archive/news-by-date/2014/pt/)

GIL, Antonio Carlos. Métodos e técnicas de pesquisa social. 6. ed. São Paulo: Atlas, 2014.

HICKEL, Denis Kern. 0 design como a expressão de fazer as coisas juntos: um entendimento ecológico. Projética, Londrina, v.6 n. ${ }^{\circ}$ 1, p. 77-96, jul. 2015. 
IBGE. Censo Agropecuário 2006: Brasil, Grandes Regiões e Unidades da Federação. Instituto Brasileiro de Geografia e Estatística, 2009. (https://ww2.ibge. gov.br/home/estatistica/economia/agropecuaria/ censoagro/2006_segunda_apuracao/default.shtm)

LAMARCHE, H. (coord.) A agricultura familiar: comparação internacional - do mito à realidade. Campinas, SP: Ed. UNICAMP, 1993.

LOCKTON, D.; HARRISON, D.; STANTON, N. The design with intent method: a design tool for influencing user behavior. Applied Ergonomics, v. 41, n. ${ }^{\circ}$ 3, pp. 382- 392, 2010.

MIOR, L. C. et al. Redes, agroindústrias familiares e os novos mercados em Santa Catarina. In: ESTEVAM, D. O.; MIOR, L. C. (Orgs). Inovações na agricultura familiar: As cooperativas descentralizadas em Santa Catarina. Florianópolis: Insular. 2014. P. 73-100.

NIEDDERER, K. et al. Creating Sustainable Innovation through Design for Behaviour Change: Full Project Report. University of Wolverhampton, Project Partners \& AHRC, 2014.

TENG, J. T. C.; SONG, S. An exploratory examination of knowledge-sharing behaviors:

solicited and voluntary. Journal of Knowledge Management, v. 15, n. ${ }^{\circ}$ 1, p. 104-117, 2011. 